ARTICLE

https://doi.org/10.1038/s41467-020-15159-2

\title{
A highly CMOS compatible hafnia-based ferroelectric diode
}

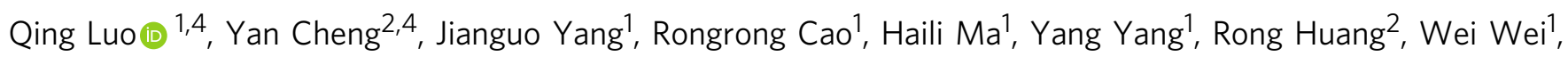
Yonghui Zheng' ${ }^{2}$, Tiancheng Gong ${ }^{1}$, Jie Yu1, Xiaoxin $\mathrm{Xu}^{1}$, Peng Yuan ${ }^{1}$, Xiaoyan $\mathrm{Li}^{1}$, Lu Tai ${ }^{1}$, Haoran Yu1, Dashan Shang (10 1, Qi Liu (i) ${ }^{1}$, Bing Yu ${ }^{3}$, Qiwei Ren ${ }^{3}$, Hangbing Lv (i) ${ }^{1 凶} \&$ Ming Liu ${ }^{1 凶}$

Memory devices with high speed and high density are highly desired to address the 'memory wall' issue. Here we demonstrated a highly scalable, three-dimensional stackable ferroelectric diode, with its rectifying polarity modulated by the polarization reversal of $\mathrm{Hf}_{0.5} \mathrm{Zr}_{0.5} \mathrm{O}_{2}$ films. By visualizing the hafnium/zirconium lattice order and oxygen lattice order with atomicresolution spherical aberration-corrected STEM, we revealed the correlation between the spontaneous polarization of $\mathrm{Hf}_{0.5} \mathrm{Zr}_{0.5} \mathrm{O}_{2}$ film and the displacement of oxygen atom, thus unambiguously identified the non-centrosymmetric $\mathrm{Pca}_{1}$ orthorhombic phase in $\mathrm{Hf}_{0.5} \mathrm{Zr}_{0.5} \mathrm{O}_{2}$ film. We further implemented this ferroelectric diode in an 8 layers 3D array. Operation speed as high as 20 ns and robust endurance of more than $10^{9}$ were demonstrated. The builtin nonlinearity of more than 100 guarantees its self-selective property that eliminates the need for external selectors to suppress the leakage current in large array. This work opens up new opportunities for future memory hierarchy evolution.

\footnotetext{
${ }^{1}$ Key Laboratory of Microelectronics Devices and Integrated Technology, Institute of Microelectronics of the Chinese Academy of Sciences, No. 3 Beitucheng West Road, Chaoyang District, Beijing 100029, China. ${ }^{2}$ Key Laboratory of Polar Materials and Devices (MOE), Department of Electronics, East China Normal University, 500 Dongchuan Road, Shanghai 200241, China. ${ }^{3}$ Xi'an UnilC Semiconductors Co., Ltd., 38 Gaoxin 6th Rd, High-Tech Industrial Development Zone, Xi'an 710075, China. ${ }^{4}$ These authors contributed equally: Qing Luo, Yan Cheng. ${ }^{凶}$ email: Ivhangbing@ime.ac.cn; liuming@ime.ac.cn
} 
$\mathrm{M}$ emory hierarchy composed of volatile and nonvolatile solid-state memories is the main building block of the computing system ${ }^{1}$. It could achieve an optimal tradeoff between performance and density in different types of memory ${ }^{2}$. However, the frequent data transfer between different memories leads to the decreased bandwidth and degraded computing efficiency, especially in the case of massive data computing, resulting in the well-known "memory wall" issue. To effectively solve the memory wall problem, a desired way is to configure a memory with high speed and high-density features.

Among various types of nonvolatile memories, ferroelectric random-access memories (FeRAM), which achieve nonvolatility by switching and sensing the polarization state of a ferroelectric capacitor, have been thought of as an excellent memory solution due to its outstanding features of low power, high speed, high endurance, and good retention. However, the scaling limitation of perovskite ferroelectric materials has hindered their widespread applications ${ }^{3}$. The development of FeRAM in semiconductor industry has been stopped at $130-\mathrm{nm}$ node for a long time $\mathrm{e}^{4,5}$. The discovery of ferroelectricity in doped $\mathrm{HfO}_{2}$ has renewed the interest in FeRAM by offering a possible solution to bridge the scaling gap between perovskite ferroelectric materials and complementary metal-oxide-semiconductor (CMOS) technology ${ }^{4,6}$.

Up to now, ferroelectric $\mathrm{HfO}_{2}$-based memories in the forms of one access transistor-one ferroelectric capacitor $(1 \mathrm{~T} 1 \mathrm{C})^{5,7}$ and ferroelectric transistor (FeFET) structures have been demonstrated, with excellent scalability down to $10-\mathrm{nm}$ node ${ }^{8}$. As shown in Supplementary Table 1, the 1T1C FeRAM requires a large capacitor area ("footprint") and undergoes destructive readout. FeFET can realize non-distructive read and 3D vertical stack ${ }^{9-11}$. However, it cannot be used as a random-access memory. On the other hand, two terminal devices, such as the ferroelectric tunneling junction (FTJ) and Fe diode, have the potential to achieve high- density crossbar array ${ }^{12-15}$. The FTJ device takes the advantage of a ferroelectric as the barrier material, and has a giant tunnel electroresistance (TER) effect by switching the ferroelectric polarization, which was generally formulated by quantum mechanical electron-tunneling mechanism. In principle, both the on and off states of FTJ obey linear or quasi-linear $I-V$ relationship, which makes it need extra selector device to diminish the sneaking current in the crossbar array. In contrast, the ferroelectric diode (Fe diode), with the working principle governed by Schottky barrier modulation as polarization reversal, has the potential to gain inherent nonlinearity and realize selector-free cross-point integration. The concept of Fe diode was first proposed in $\mathrm{PbTiO}_{3}$ perovskite thin films ${ }^{16}$ and later in bulk $\mathrm{BiFeO}_{3}$ single crystals ${ }^{17}$. The ultralow readout currents in the orders of $20 \mathrm{~mA} / \mathrm{cm}^{2}$ and low on/off ratio limited their miniaturization. In 2011, Jiang et al. reported a Fe-diode current density up to $5.4 \mathrm{~A} /$ $\mathrm{cm}^{2}$ in $\mathrm{Pt} / \mathrm{BiFeO}_{3} / \mathrm{SrRuO}_{3}$ thin-film capacitors by a plate-like growth mode ${ }^{18}$. However, it is still too low to be detected by a periphery circuit in chip. Meanwhile, the CMOS- incompatible film growth technique makes the current state of the art of $\mathrm{Fe}$ diode unable to be implemented in the 3D structure.

Here, we proposed a hafnia-based switchable Fe diode by spontaneous polarization reversal in the non-centrosymmetric $\mathrm{Pca}_{1}$ orthorhombic phase. First, the non-centrosymmetric orthorhombic phase $\left(\mathrm{Pca}_{1}\right)$ in $\mathrm{Hf}_{0.5} \mathrm{Zr}_{0.5} \mathrm{O}_{2}$ (HZO) film was unambiguously identified by visualizing both the hafnium/zirconium lattice order and oxygen lattice order with atomicresolution spherical aberration (Cs)-corrected scanning transmission electron microscopy (STEM). The displacement of oxygen atoms was ascribed as the root of the spontaneous polarization that occurred in the domain. Then, based on this promising ferroelectric material, we demonstrated a fully CMOS-compatible Fe diode with high readout current density

$\left(>200 \mathrm{~A} / \mathrm{cm}^{2}\right)$ and built-in nonlinearity $(>100)$, which was further 3D integrated up to eight layers with vertical size down to $20 \mathrm{~nm}$. High operation speed of $20 \mathrm{~ns}$ and good endurance properties were achieved, showing its promising prospect to achieve high speed and high density. This work opens up new opportunities for future memory hierarchy evolution in the computing system.

\section{Results}

Identification of the non-centrosymmetric $\mathrm{Pca}_{1}$ phase. Metal-ferroelectric-metal (MFM) capacitors with HZO film (thickness of $10 \mathrm{~nm}$ ) were fabricated and characterized to confirm its ferroelectricity. The detailed material deposition and device fabrication processes were described in the "Experimental" section and Supplementary Fig. 1. To elucidate the FE properties of $\mathrm{TiN} / \mathrm{HZO} / \mathrm{TiN}$ samples, the measurements were performed using the PUND (positive-up negative-down) technique ${ }^{19}$. Figure $1 \mathrm{a}$ is the typical hysteresis $\mathrm{P}-\mathrm{V}$ loop of TiN/HZO/TiN device after $400^{\circ} \mathrm{C}$ of rapid thermal annealing, showing a remnant polarization $(\mathrm{Pr})$ of $\sim 17 \mu \mathrm{C} / \mathrm{cm}^{2}$ and coercive field of $-1.4 \mathrm{MV} / \mathrm{cm}$ and $+1.9 \mathrm{MV} / \mathrm{cm}$. Figure $1 \mathrm{~b}$ depicts the bright-field (BF) STEM image of TiN/HZO/TiN stack, in which the polycrystalline nature of the $\mathrm{HZO}$ thin film as well as the boundaries with TiN metal electrodes are visible. In the close-up image of the HZO film (Fig. 1c), $\mathrm{o}-$ and $\mathrm{m}$-phases can be identified in certain directions because the $\mathrm{m}$-phase has a non-vertical $\beta$ angle. Based on the apparent relative angle differences between the two corresponding lattice

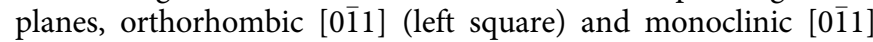
grains (right square) were clearly identified, indicating the coexistence of the two phases in the HZO film.

It is generally accepted that the orthorhombic phase (o-phase) is the origin of ferroelectricity ${ }^{6,20,21}$. However, there are four different space groups in the o-phase, i.e., $\mathrm{Pca} 2_{1}, \mathrm{Pbcm}, \mathrm{Pmn} 2_{1}$, and Pbca, among which, only the $\mathrm{Pca} 2_{1}$ and $\mathrm{Pmn} 2_{1}$ space groups

a

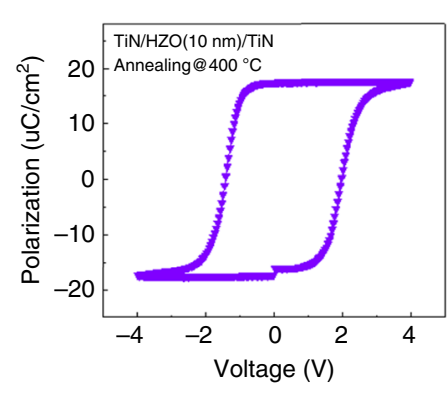

C

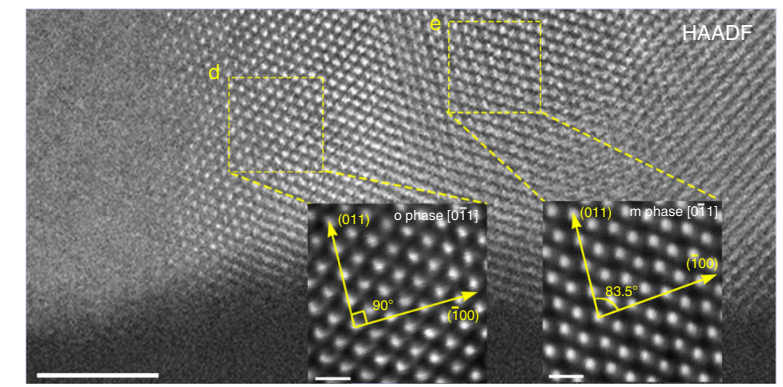

Fig. 1 Planar metal-ferroelectric-metal (MFM) capacitors. a Typical P-V loop $\left(2.5 \mathrm{kHz}\right.$ ) of the $\mathrm{Hf}_{0.5} \mathrm{Zr}_{0.5} \mathrm{O}_{2}$ (HZO) film derived from positive-up negative-down (PUND) measurements. b STEM-BF image of the TiN/ HZO/TiN stack. Scale bars, $10 \mathrm{~nm}$. c STEM-HAADF image of the HZO thin film with two specific crystal grains, projected along o [011] (left square) and $\mathrm{m}$ [0111] (right square) zone axes. Scale bars, $5 \mathrm{~nm}$. Scale bar inset, $0.5 \mathrm{~nm}$. 
are the polar phase. The Pmn $2_{1}$ phase could be easily ruled out by the image of the hafnium sublattice. However, distinguishing between the $\mathrm{Pbca}, \mathrm{Pbcm}$, and $\mathrm{Pca} 2_{1}$ space groups is quite challenging, which could only be realized by visualizing the tiny difference of oxygen sublattice.

Sang et al. ${ }^{21}$ reported the presence of the o-phase in Gd-doped $\mathrm{HfO}_{2}$ films by using the atomic-resolution STEM high-angle annular dark-field (HAADF) technique, in which the projected hafnium sublattices are in good agreement with the o-lattice. Further, in order to distinguish the different space groups of the o-phase, they showed the position-averaged convergent beam electron diffraction $\left(\mathrm{PACBED}^{22}\right)$ patterns from a certain o-grain that lacks mirror symmetry, to imply the existence of noncentrosymmetric orthorhombic Pca2 1 structure. However, there is no directly convincing evidence to distinguish the different ospace groups and elucidate the origin of the noncentrosymmetry of $\mathrm{Pca} 2_{1}$. Only the oxygen displacement along the polarization direction is maximum; the visible shift in atomic scale has never been experimentally reported. Hence, solid evidences on shortrange ordering of the oxygen sublattice and displacement in the local domain are essentially required.

In order to elucidate the ferroelectric nature of the orthorhombic phase, the sublattice order of hafnium/zirconium and oxygen atoms was directly detected by using the atomic STEMHAADF and ABF technique. For HZO lattice, only one direction can maximize the difference in oxygen atomic displacement to identify the different o-phase space groups. Here, [010] is the most suitable zone axis to project the atom columns. It can distinguish monoclinic, orthorhombic, and tetragonal phases, and it is also relatively easily to tell the differences in the oxygen atom arrangement, as shown in Supplementary Fig. 2. Figure 2a is a typical atomic STEM-HAADF image of the HZO o-phase, projected along the [010] zone axes, which fits well with the simulated result (inset) using the $\mathrm{Pca}_{2}$ space group. In this HAADF image, only heavy atoms such as $\mathrm{Hf}$ and $\mathrm{Zr}$ can be identified, which have very similar arrangement ${ }^{21}$ in different space groups of the o-phase. Further, atomic-resolution STEM$\mathrm{ABF}$ technique, which is sensitive to light elements, was used to inspect the information of oxygen atoms. Figure $2 \mathrm{~b}$ is the corresponding $\mathrm{ABF}$ experimental image of the o-phase projected along the [010] zone axes. It includes the inset of the simulated result and the overlaid model using the o-phase with $\mathrm{Pca} 2_{1}$ space group. The shift of oxygen atoms is indicated by purple arrows. The experimental and simulated results are completely consistent, intuitively confirming the existence of non-centrosymmetric $\mathrm{Pca} 2{ }_{1}$ structure in the hafnia ferroelectric material. In addition, Supplementary Fig. 3 gives the simulated ABF pictures of the ophase with $\mathrm{Pbcm}$ and $\mathrm{Pbca}$ space groups, which are totally different from the experimental result presented in Fig. 2b. Figure $2 \mathrm{c}$ is the schematic of the $\mathrm{HZO}$ unit cell, in which, two kinds of oxygen $\left(\mathrm{O}_{\mathrm{I}}, \mathrm{O}_{\mathrm{II}}\right)$ with different displacement behavior were denoted, and $D_{\mathrm{O}}$ is the shift distance of the oxygen atoms. Afterward, the oxygen atomic displacement vector map (distance and direction) is obtained, as shown in Fig. $2 \mathrm{~d}$ by quantifying the $\mathrm{ABF}$ image of Fig. $2 \mathrm{~b}$ (the calculation process was described in Supplementary Fig. 4), with the yellow arrows indicating the reversed oxygen atom displacement $\left(D_{\mathrm{O}}\right)$ vectors. Here, the $\mathrm{O}_{\mathrm{II}}$ site oxygen columns significantly shift $(\sim 13.5 \%$ of the c-lattice parameter $^{23}$ ) from the central position of the four nearest heavy atoms, while the $\mathrm{O}_{\text {I }}$ site oxygen columns shift slightly $(<1 \%$ of the c-lattice parameter $\left.{ }^{23}\right)$. The different displacements of the oxygen columns are attributed to their different coordination number, that is, the fourfold $\mathrm{O}_{\mathrm{I}}$ site oxygen in constant position represents paraelectric behavior, while the shift of threefold $\mathrm{O}_{\mathrm{II}}$ site oxygen is a

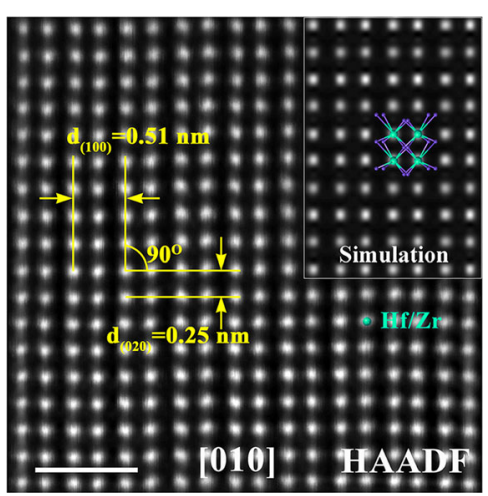

b

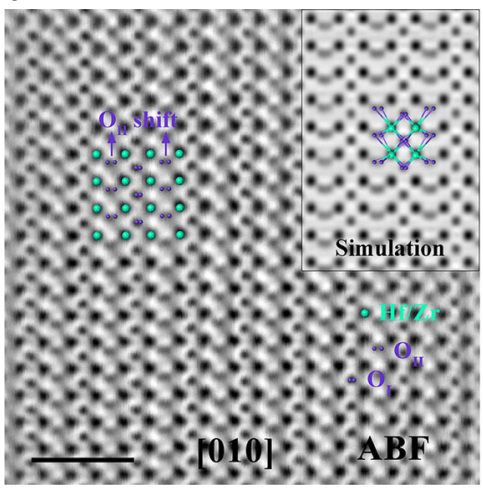

e
C

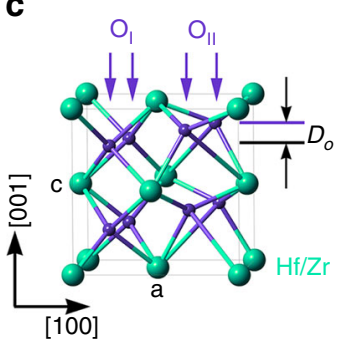

d

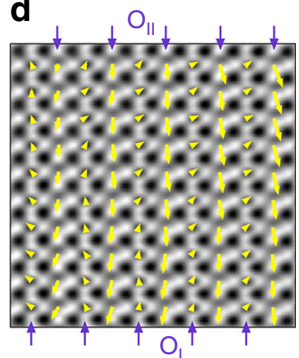

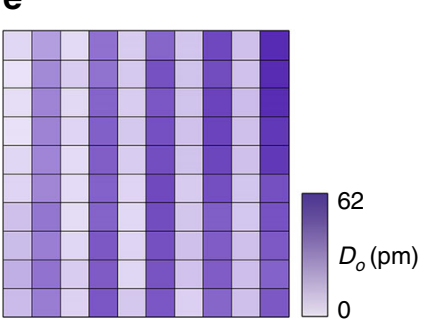

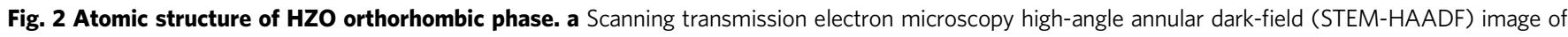
the $\mathrm{HZO}$ grain projected along $\mathrm{O}$ [010] zone axes with the inset of simulated HAADF picture. Scale bar, $1 \mathrm{~nm}$. b The corresponding scanning transmission electron microscopy high-angle annular dark-field annular bright-field (STEM-ABF) image of (a) with the inset of simulated ABF picture. Scale bar, 1 nm. c Schematic of the unit cell of $\mathrm{HZO}$, where $D_{\mathrm{O}}$ denotes the relative displacement of $\mathrm{O}$ atoms. d $\mathrm{O}$ atomic displacement vector map from the area in (b). The yellow arrows indicate reversed $\mathrm{O}$ atom displacement $\left(D_{\mathrm{O}}\right)$ vectors, which were consistent with the spontaneous polarization direction in (b). e Twodimensional $\mathrm{O}$ atomic displacement map relative to the central positions of the four nearest heavy atoms, which corresponds to the yellow arrows in (d). 
the origin of ferroelectricity from the microscopic view (see Supplementary Fig. 5). Hence, the visualized map from the above two-dimensional oxygen displacement vectors is illustrated in Fig. 2e. The periodic dark- and light-purple columns on the map fit well with the asymmetric oxygen shift in $\mathrm{Pca}_{1}$ structure. These results visualize the oxygen configuration by using Cs-corrected STEM-ABF technique, which could deeply probe into the intrinsic nature of the ferroelectric o-phase in hafnium/zirconium oxides.

Switchable Fe diode and 3D integration. Based on such a thin $(\sim 10-\mathrm{nm})$ ferroelectric layer (HZO), a switchable Fe-diode device was demonstrated, and the fabrication process is shown in Supplementary Fig. 6. Figure 3 a shows the schematic structure of the TiN/HZO/TiN Fe diode, with Schottky contacts formed at the interfaces between the metal electrodes and ferroelectric films. The positive polarization charges can be neutralized by electrons and then induce the accumulation of electrons in the polarization head side, leading to the reduction of the barrier height; the negative polarization charges can be compensated by oxygen vacancies with positive charge, resulting in upward-band bending. Thus, the Schottky-to-ohmic contact was formed in the TiN/ $\mathrm{HZO} / \mathrm{TiN}$ device (Fig. 3b), leading to a diode-like conduction. As the polarization switched, the direction of the diode was changed with the direction of polarization. Figure $3 \mathrm{c}$ shows the $I-V$ characteristics of the Fe diode device (the whole $I-V$ curve is shown in Supplementary Fig. 7). After sweeping to positive voltage, the current changes from low to high, and the state changes from negative-forward diode to positive-forward diode (blue lines). Similar behavior can be observed in negative voltage sweeping (red lines), where the state changes from positiveforward diode to negative-forward diode. Due to the existence of Schottky barrier between HZO and electrode, this Fe diode exhibits inherent nonlinearity (more than 100), defined as the ratio of the current read at $\mathrm{Vr}$ and $\mathrm{Vr} / 2$. This attribute is important for suppressing the leakage current in a cross-point array, eliminating the necessity of adding an external selector device in series with each memory cell ${ }^{24-26}$. Read disturbance is a common issue of two-terminal devices, especially the ferroelectric devices due to its accumulative switching of polarization ${ }^{27}$. In the case of polarization state with the same direction of read voltage polarity (e.g., $+2 \mathrm{~V} / 100 \mathrm{~ns}$ ), the read operation has negligible influence on the polarization state (polarization-down, on state) (shown in Supplementary Fig. 8a). However, in the case of polarization state (polarization-down, off state) opposite to the polarity of read voltage, the read pulse will gradually increase the off-state current, leading to disturbance issue. This issue could be solved by a combined pulse read scheme with a pair of positive and negative read pulses. The reverse pulse is helpful to restore the original polarization state. As shown in Supplementary Fig. 8b, by using this method, the off-state current remains almost unchanged after $10^{9}$ read pulses.

In order to verify the potential of high-density integration, the HZO-based $\mathrm{Fe}$ diode was further implemented in a 3D architecture. Figure 4a shows the schematic view of the 8-layer stacked Fe diode array. The detailed process flow is shown in Supplementary Fig. 9. Figure $4 \mathrm{~b}$ shows the TEM image of the 8layer vertical structure and the element mapping in the selected zone. The magnified device area is shown in Fig. 4c. TiN/HZO/ $\mathrm{TiN} / \mathrm{W}$ device structure and the interface in between could be clearly observed. Figure $4 \mathrm{~d}$ shows the $I-V$ curves of the Fe-diode devices in the $4 \times 8$ array framed that was given in Fig. 4 a. Each cell in the eight-layer vertical structure has similar $I-V$ curves. The typical $I-V$ characteristics of $\mathrm{Fe}-\mathrm{HfO}_{2}$ diodes are shown in Fig. 4e, with the numerical symbols denoting the sequence of voltage sweeps. This $I-V$ curve has similar diode-switching behavior as the one shown in Fig. 3c, with forward rectifying (blue curve) occurring due to positive polarization and reversal rectifying (read curve) occurring due to negative polarization. Different memory states could be read out easily by applying a proper read voltage (e.g., $2 \mathrm{~V}$ ). Large on/off ratio of more than $10^{4}$ was realized. The current density is as high as $\sim 200 \mathrm{~A} / \mathrm{cm}^{2}$, which is more than 40 times of the traditional $\mathrm{Fe} \operatorname{diode}^{18}$. This high current density could make the memory status be detected easily by using the sense amplifiers in the peripheral circuitry. To confirm the high sensing speed, we evaluated the sensing time by using a current-sampling-based sense amplifier (SA) (Supplementary Fig. 10). The simulation was carried out for read lowresistance state $\left(I_{\text {cell }}=100 \mathrm{nA}\right)$ and high resistance state $\left(I_{\text {cell }}=\right.$ $5 \mathrm{nA}$ ) with 128 cells per BL (Supplementary Figs. 11 and 12). It can be seen that the CSA can sense both high- and low-resistance states within $65 \mathrm{~ns}$, and the margin is larger than $60 \mathrm{mV}$. By further decreasing the HZO film thickness, lower switching voltages and higher operation current could be obtained (Supplementary Fig. 13). Considering the peripheral circuit design in a memory chip, the sensing current and voltage window should be high enough to guarantee the successful readand-write operation; comprise should be made by selecting the proper material thickness.

Both HRS and LRS show good nonvolatility and stability, confirmed by the retention test over $50,000 \mathrm{~s}$ at $85^{\circ} \mathrm{C}$ (shown in Supplementary Fig. 14). To evaluate the array-level performance of the Fe diode device, statistical measurement was carried out on an $8 \times 8 \times 323 \mathrm{D}$ array. The corresponding resistance distributions of HRS and LRS reads at $2 \mathrm{~V}$ are illustrated in Fig. 4f, showing good uniformity with the notably on/off ratio $\left(\sim 10^{4}\right)$. By purely using the Schottky emission equation with the barrier height varied with ferroelectric polarization, the theoretical on/off ratio $\left(>10^{5}\right)$ is larger than the measured one $\left(>10^{4}\right)$, implying that other conduction mechanisms might be dominant in the OFF state (Supplementary Note 1), such as the trap-assisted tunneling, as found in most of the transition metal oxides $27-31$. Figure $4 \mathrm{~g}$ displays the distributions of the nonlinearity of 100 cells in the 2$\mathrm{kb}$ array, confirming excellent uniformity in the low-resistance state and outstanding self-selective characteristics. To confirm the large-scale feasibility, read-and-write margins were analyzed based on the device specifications (Supplementary Fig. 15). Owing to its high nonlinearity and on/off ratio, a sufficient read margin (10\%) can be obtained for up to $100-\mathrm{kb}$ array in the worstcase (only one BL pulled up and all unselected cells at LRS) ${ }^{32}$ condition. It is not sufficient enough to build large-size array, and further optimization is required to improve the nonlinearity. These aforementioned results suggest that the Fe-diode device has high potential to be used for high-density 3D memory application.

Ultrahigh speed and high endurance. The switching speed of the Fe diode was measured by using voltage pulse stimulus. We define the forward-diode switching as the SET process and reverse-diode switching as the RESET process. We altered the voltage pulses from a 20 -ns to 5 - $\mu$ s width with a magnitude from 3 to $11 \mathrm{~V}$. The ultrahigh operation speed of the Fe diode memory was attributed to the quick polarization flipping of the $\mathrm{HZO}$ film, which has been indicated to be able to switch in $<20$ ns by experimental verification ${ }^{33}$. Figure 5 a shows the voltage dependence of the SET and RESET operating speed for Fe diode. Each data point was the mean value of the operation voltage with different pulse widths in Supplementary Fig. 16, showing a compromise between the speed and voltage height. Figure $5 b$ shows the endurance test result under successive voltage pulses with $9.5 \mathrm{~V} / 20 \mathrm{~ns}$ for SET and $5.9 \mathrm{~V} / 20 \mathrm{~ns}$ for RESET 

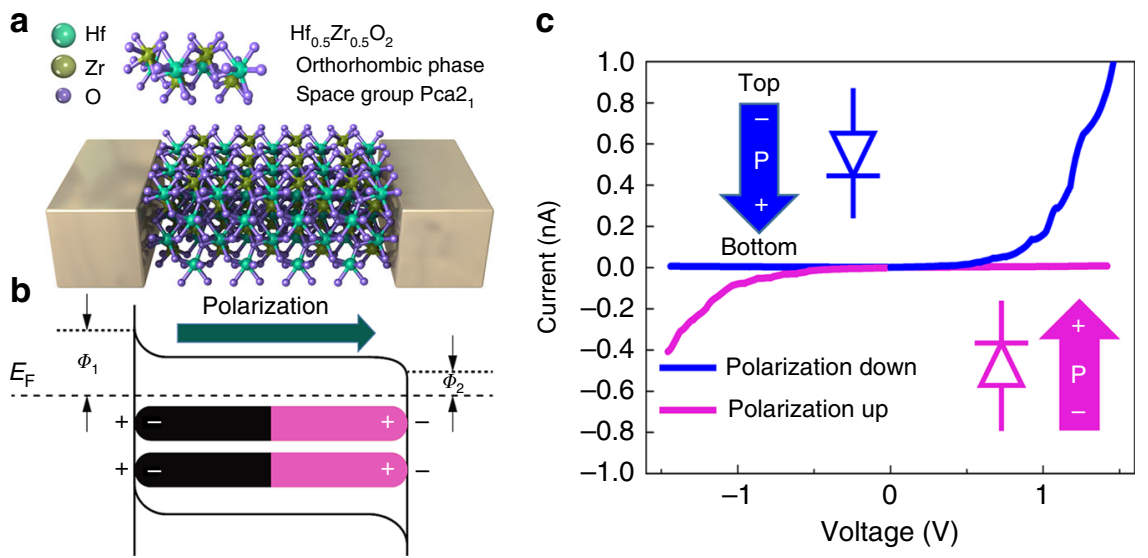

Fig. 3 Ferroelectric diode with a thin ( $10-\mathbf{n m})$ ferroelectric layer. a Schematic structure of the ferroelectric diode. b Schematics of the energy band diagrams of the Schottky-to-ohmic interfacial contacts in TiN/HZO/TiN modulated by polarization orientations. c Nonlinear diode-like I-V of the TiN/ $\mathrm{HZO} / \mathrm{TiN}$ device with a cell size of $1 \mu \mathrm{m}$. Insets represent a schematic of the potential energy profiles in two opposite polarization states.

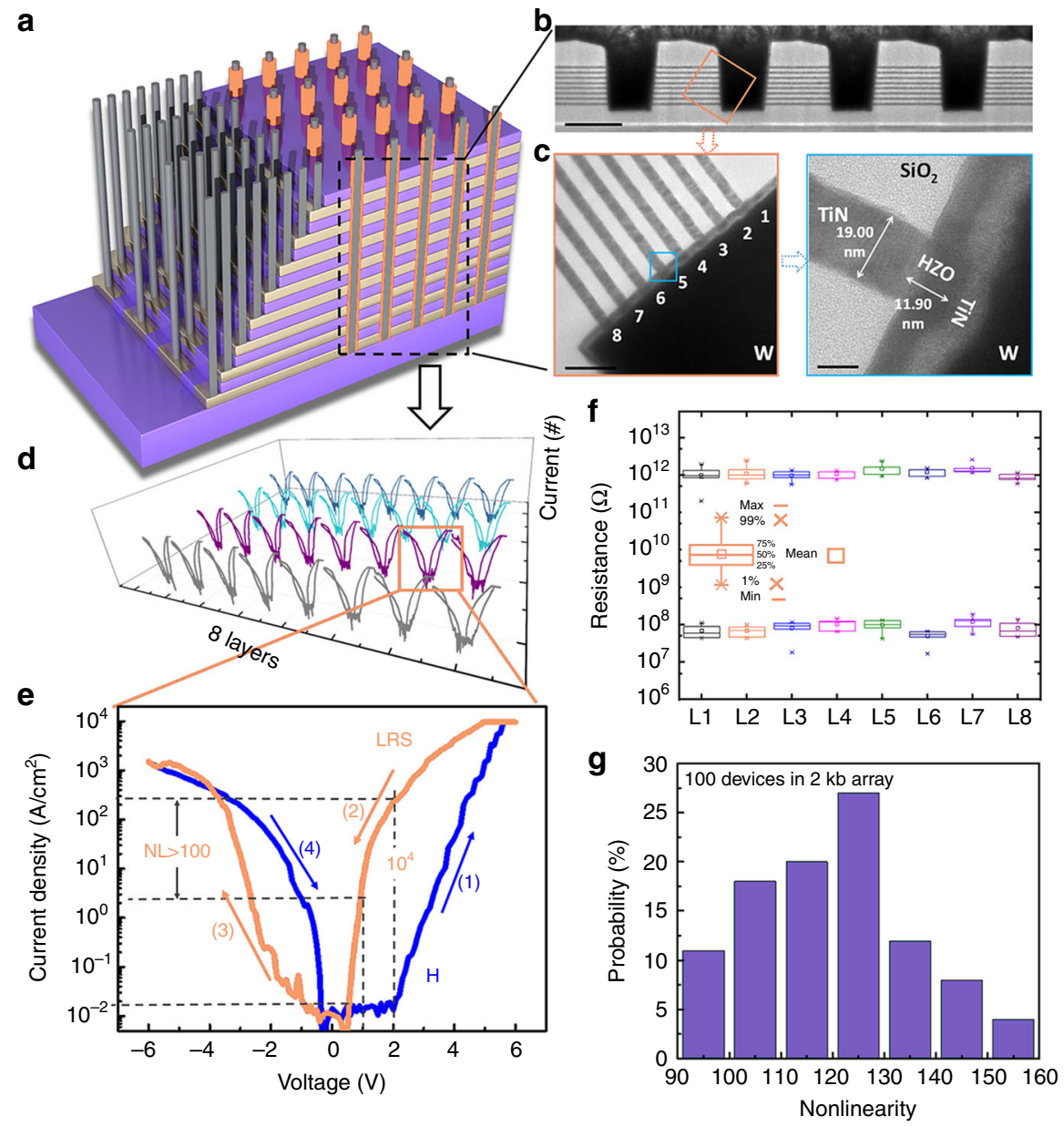

Fig. 4 High-density 3D integration of the ferroelectric diode devices. a A schematic view of the 8-layer 3D vertical ferroelectric diode (Fe diode) array. b Cross section of the 3D vertical structure with Fe diode devices and the detailed structure information for the devices, where a 417.5 -nm hole structure and 8-layer vertical memory cells can be observed clearly. Scale bar, $500 \mathrm{~nm}$. c The cell size was defined by the thickness of the TiN and the perimeter of the holes $(19 \mathrm{~nm} \times 1.31 \mu \mathrm{m})$. Scale bar (left), $10 \mathrm{~nm}$. Scale bar (right), $10 \mathrm{~nm}$. d $/-V$ curves of the Fe diode devices in the $4 \times 8$ array framed in (a). e Typical $I-V$ curve of the $\mathrm{TiN} / \mathrm{Hf}_{0.5} \mathrm{Z}_{0.5} \mathrm{rO}_{2} / \mathrm{TiN} / \mathrm{W}$ device. Switchable diode property was achieved. In this device, low operation current $(<1 \mu \mathrm{A})$ and high nonlinearity $(>100)$ were achieved. $\mathbf{f}$ Resistance distributions of the switchable diode device in the 8-layer array. $\mathbf{g}$ Distribution of the nonlinearity in 3D array. 

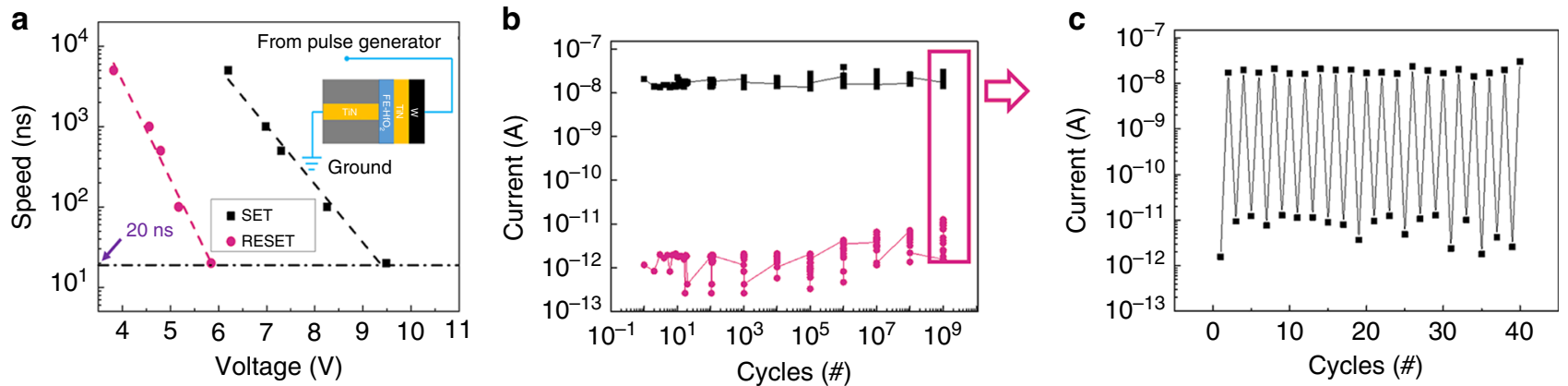

Fig. 5 Ultrahigh speed and high endurance. a Voltage dependence of the SET and RESET operation speed for Fe-diode memory. The inset shows a schematic of the device structure with the pulse signal. $\mathbf{b}$ Endurance test. The device can switch $>10^{9}$ pulse cycles. c For each order of the cycling number, 20 cycles of write-and-read operation were carried out to confirm the effectiveness of the write pulses.

Table 1 Comparison of the various reported Fe-diode devices.

\begin{tabular}{|c|c|c|c|c|c|c|}
\hline Structure & Substrate & Preparation method & Thickness of Fe material & Current density & Nonlinearity & On/off ratio \\
\hline $\mathrm{Au} / \mathrm{PbTiO}_{3} / \mathrm{LSC}^{16}$ & $\mathrm{LaA1O}_{3}$ & Epitaxial deposition & $200 \mathrm{~nm}$ & $0.1 \mathrm{~A} / \mathrm{cm}^{2}$ & 2 & 2 \\
\hline $\mathrm{PZT} /(\mathrm{LSMO})^{38}$ & $\mathrm{SrTiO}_{3}$ & PLD & $30 \mathrm{~nm}$ & $0.1 \mathrm{~A} / \mathrm{cm}^{2}$ & 2 & 300 \\
\hline $\mathrm{Ag} / \mathrm{BFO} / \mathrm{Ag}^{17}$ & / & / & 90 um & $20 \mathrm{~mA} / \mathrm{cm}^{2}$ & 5 & 20 \\
\hline $\mathrm{SRO} / \mathrm{PZT} / \mathrm{SRO} 40$ & $\mathrm{SrTiO}_{3}$ & PLD & $150 \mathrm{~nm}$ & $10 \mathrm{~mA} / \mathrm{cm}^{2}$ & 2 & 4 \\
\hline $\mathrm{SRO} / \mathrm{BFO} / \mathrm{Pt}^{18}$ & $\mathrm{SrTiO}_{3}$ & PLD & $120 \mathrm{~nm}$ & $5.4 \mathrm{~A} / \mathrm{cm}^{2}$ & 5 & 4 \\
\hline $\mathrm{SRO} / \mathrm{BFO} / \mathrm{Pt}^{41}$ & $\mathrm{SrTiO}_{3}$ & PLD & $40 \mathrm{~nm}$ & - & 2 & 753 \\
\hline
\end{tabular}

(Supplementary Fig. 17). After $10^{9}$ cycles, the on-/off-state ratio read by a $2-\mathrm{V}$ voltage pulse can still be well maintained. For each order of the cycling number, 20 cycles of write-and-read operation were carried out to confirm the effectiveness of the write pulses (Fig. 5c). The pulse test results show that this Fe-diode device has robust cycling capability with fast operation speed.

\section{Discussion}

The performance comparison between the HZO-based Fe diode and the perovskite material-based Fe diode is shown in Table 1. Outstanding properties, such as better CMOS compatibility, higher scalability, and higher current density, were achieved in HZO- based Fe diode. Together with its 3D integration capability, the HZO-based Fe diode shows high possibility of combining high speed and high-density features. Thus, complex memory hierarchy composed of working and storage memory is constructed and forms the main building block of the computing system. However, the performance gap between the working and storage memory becomes the bottleneck for system performance improvement, especially in the case of massive data computing, and results in the well-known "memory wall" issue. There is an urgent demand to find a new memory solution with both the merit of high speed and high density. The Fe diode (Supplementary Table 2) demonstrated here shows promising propects on providing unified performance for memory hierachy evolution in the future.

In summary, a 3D-stackable Fe-diode device with high speed and high-density memory characteristics was demonstrated. With the help of polarization reversal, the Fe diode exhibited switchable rectifying behavior. The correlation between the spontaneous polarization and short-range order displacement of the oxygen atom was clearly revealed, which paved a new way to understand the fundamental ferroelectric theory of the hafina-based materials, and figure out effective approaches to optimize the device performance. The demonstration of 3D vertical integration up to 8 layers shows its good potential to achieve high-density storage. Together with the high speed and high-endurance characteristics, this $\mathrm{Fe}$-diode device opens up new opportunities for future memory and storage convergence.

\section{Methods}

Experimental. (1) Fabrication of planar metal-ferroelectric-metal (MFM) capacitors: Planar MFM capacitors were fabricated on p-doped Si (100) substrates. Initially, TiN bottom electrodes (BEs) of 30-nm thickness were formed by physical vapor deposition (PVD). Thermal ALD at $260^{\circ} \mathrm{C}$ was then used to deposit $\mathrm{Zr}$ doped $\mathrm{HfO}_{2}$ films from $\mathrm{Hf}\left[\mathrm{N}\left(\mathrm{C}_{2} \mathrm{H}_{5}\right) \mathrm{CH}_{3}\right]_{4}$ and $\mathrm{Zr}\left[\mathrm{N}\left(\mathrm{C}_{2} \mathrm{H}_{5}\right) \mathrm{CH}_{3}\right]_{4}$. An ALD cycle ratio of 1:1 (Hf-to- $\mathrm{Zr}$ precursor pulses) was applied to achieve a $\mathrm{Zr}$ content of $50 \%$ (cationic ratio of $\mathrm{Zr} /[\mathrm{Zr}+\mathrm{Hf}]$ ) in the $\mathrm{HfO}_{2}$ layers. Similar to the $\mathrm{BEs}$, 30-nm-thick TiN top electrodes (TEs) were deposited by PVD. The previously amorphous $\mathrm{Zr}$ : $\mathrm{HfO}_{2}$ films were then crystallized by rapid thermal annealing in an $\mathrm{N}_{2}$ ambient at $400{ }^{\circ} \mathrm{C}$ for $30 \mathrm{~s}$. The top electron was etched for PFM testing. $\mathrm{SiO}_{2}$ thin film was deposited on the HZO film. After optical lithography and etch process, a hole etched down to the HZO film with a diameter of $2 \mu \mathrm{m}$ was formed. TiN top electron was deposited by ALD; the cell size of Fe diode was defined by the area of contact between TiN and HZO. (2) 3D integration of Fe diode: Preparation of the 8-layer 3D vertical memory with Fe-diode cells: First, multiple TiN $(20 \mathrm{~nm}) / \mathrm{SiO}_{2}$ (30-nm) layers were deposited by PVD and PECVD, respectively. Patterning and only one-step etching were applied to form stacked wordlines $(\mathrm{WL})$ with a smooth sidewall profile. After $\mathrm{SiO}_{2}$ filling in the trench, a $500-\mathrm{nm}$ hole is etched down to the bottom $\mathrm{SiO}_{2}$. $\mathrm{Hf}_{0.5} \mathrm{Zr}_{0.5} \mathrm{O}_{2}$ bilayer was deposited on the sidewall sequentially by ALD, followed by depositing of TiN/W by the sputtering to fill the hole as the pillar electrode (BL). Each horizontal WL was opened by selective etching successively. The area of the memory cell was defined by the thickness of the bottom electrode $\operatorname{TiN}(20 \mathrm{~nm})$ and the perimeter of the hole.

Characterization. The DC and pulse endurance of a self-selective cell were tested by an Agilent B1500A semiconductor parameter analyzer connected to the experimental device. The pulse measurements were performed using the HVSPGU module of Agilent B1500A. During the electrical measurement, the W top electrode was biased, while the TiN bottom electrode was grounded.

STEM part. The annealed TiN/HZO/TiN film was fabricated into a cross-section sample by using focused ion beam (FIB) technique in FEI Helios G4 system, including low-pressure polishing process at 5 and $2 \mathrm{keV}$, and then cleaning in 
Gatan 691 PIPS at $1-0.5 \mathrm{keV}$ for removing the residual contamination and possible damage. The scanning transmission electron microscopy (STEM) analysis was carried out on JEM Grand ARM300F microscope operated at $300 \mathrm{kV}$ using STEM mode with probe corrector, including high-angle annular dark-field (HAADF), bright-field $(\mathrm{BF})$, and annular bright-field ( $\mathrm{ABF}$ ) images with a probe convergence semiangle of $\sim 18 \mathrm{mrad}$. The STEM-HAADF images were taken by an annular dark-field image detector with the inner semiangle larger than $64 \mathrm{mrad}$. The STEM-ABF images were taken by a BF image detector with the central part being blocked by a beam stopper, in which, the collection semiangle is $12-24 \mathrm{mrad}$.

STEM simulation part. We use the software OSTEM $^{34}$ with multislice method to simulate the STEM-ABF and STEM-HAADF images of $\mathrm{Pca} 2_{1}$ and $\mathrm{Pbcm}$ phases; the crystal structures are from CIF files in the literature ${ }^{34-37}$. The thickness of the sample is set to about $20 \mathrm{~nm}$. The microscope parameters are high voltage $300 \mathrm{kV}$, $\mathrm{Cc}=1 \mathrm{~mm}$, convergence angle $18 \mathrm{mrad}$, defocus $0 \mathrm{~mm}$, spherical aberr. $\mathrm{C} 3=$ $0.005 \mathrm{~mm}$, and temperature $300 \mathrm{~K}$. Two detectors are used for the HAADF and $\mathrm{ABF}$, with the inner-to-outer angles $40-200 \mathrm{mrad}$ and $12-24 \mathrm{mrad}$, respectively. The simulation is along the [010] zone axes, and a series of images tilted from zone axes are also carried out to estimate the effect of tilt to the experimental results.

\section{Data availability}

All data needed to evaluate the conclusions are present in the paper and/or the Supplementary Materials. Additional data related to this paper may be requested from the authors.

Received: 30 August 2019; Accepted: 19 February 2020; Published online: 13 March 2020

\section{References}

1. Wong, H.-S. P. \& Salahuddin, S. Memory leads the way to better computing. Nat. Nanotechnol. 10, 191 (2015).

2. Kent, A. D. \& Worledge, D. C. A new spin on magnetic memories. Nat. Nanotechnol. 10, 187 (2015).

3. Park, M. H. et al. Ferroelectricity and antiferroelectricity of doped thin $\mathrm{HfO}_{2}-$ based films. Adv. Mater. 27, 1811-1831 (2015).

4. Müller, J. et al. Ferroelectricity in $\mathrm{HfO}_{2}$ enables nonvolatile data storage in $28 \mathrm{~nm}$ HKMG. in 2012 Symposium on VLSI Technology (VLSIT) 25-26 (2012).

5. Müller, J. et al. Ferroelectric hafnium oxide: a CMOS-compatible and highly scalable approach to future ferroelectric memories. in 2013 IEEE International Electron Devices Meeting (IEDM) 10.18.1-10.18.14 (2013).

6. Böscke, T. S., Müller, J., Bräuhaus, D., Schröder, U. \& Böttger, U. Ferroelectricity in hafnium oxide thin films. Appl. Phys. Lett. 99, 102903 (2011).

7. Polakowski, P. et al. Ferroelectric deep trench capacitors based on $\mathrm{Al}: \mathrm{HfO}_{2}$ for 3D nonvolatile memory applications. in 2014 IEEE 6th International Memory Workshop (IMW) 1-4 (2014).

8. Luo, Q. et al. Hybrid 1T e-DRAM and e-NVM realized in One $10 \mathrm{~nm}$ node Ferro FinFET device with charge trapping and domain switching effects. in 2018 IEEE International Electron Devices Meeting (IEDM) 2.6.1-2.6.4 (2018).

9. Florent, K. et al. First demonstration of vertically stacked ferroelectric Al doped $\mathrm{HfO}_{2}$ devices for NAND applications. in 2017 Symposium on VLSI Technology T158-T159 (2017).

10. Florent, K. et al. Vertical ferroelectric $\mathrm{HfO}_{2}$ FET based on 3-D NAND architecture: towards dense low-power memory. in 2018 IEEE International Electron Devices Meeting (IEDM) 2.5.1-2.5.4 (2018).

11. Pesic, M. et al. How to make DRAM non-volatile? Anti-ferroelectrics: a new paradigm for universal memories. in 2016 IEEE International Electron Devices Meeting (IEDM) 11.16.11-11.16.14 (2016).

12. Wang, H. et al. Direct observation of room-temperature out-of-plane ferroelectricity and tunneling electroresistance at the two-dimensional limit. Nat. Commun. 9, 3319 (2018).

13. Mo, F., Tagawa, Y., Saraya, T., Hiramoto, T. \& Kobayashi, M. Scalability study on ferroelectric- $\mathrm{HfO}_{2}$ tunnel junction memory based on non-equilibrium Green function method with self-consistent potential. in 2018 IEEE International Electron Devices Meeting (IEDM) 16.13.11-16.13.14 (2018).

14. Max, B., Hoffmann, M., Slesazeck, S. \& Mikolajick, T. Ferroelectric tunnel junctions based on ferroelectric-dielectric $\mathrm{Hf}_{0.5} \mathrm{Zr}_{0.5} \mathrm{O}_{2} / \mathrm{Al}_{2} \mathrm{O}_{3}$ capacitor stacks. in 2018 48th European Solid-State Device Research Conference (ESSDERC) 142-145 (2018).

15. Max, B., Hoffmann, M., Slesazeck, S. \& Mikolajick, T. Direct correlation of ferroelectric properties and memory characteristics in ferroelectric tunnel junctions. IEEE J. Electron. Devi. 7, 1175-1181 (2019).

16. Blom, P. W. M., Wolf, R. M., Cillessen, J. F. M. \& Krijn, M. P. C. M. Ferroelectric Schottky diode. Phys. Rev. Lett. 73, 2107-2110 (1994).
17. Choi, T., Lee, S., Choi, Y., Kiryukhin, V. \& Cheong, S.-W. Switchable ferroelectric diode and photovoltaic effect in $\mathrm{BiFeO}_{3}$. Science 324, 63-66 (2009).

18. Jiang, A. Q. et al. A resistive memory in semiconducting $\mathrm{BiFeO}_{3}$ thin-film capacitors. Adv. mater. 23, 1277-1281 (2011).

19. Scott, J. F. et al. Switching kinetics of lead zirconate titanate submicron thinfilm memories. J. Appl. Phys. 64, 787-792 (1988).

20. Müller, J. et al. Ferroelectricity in Simple Binary $\mathrm{ZrO}_{2}$ and $\mathrm{HfO}_{2}$. Nano Lett. 12, 4318-4323 (2012).

21. Sang, X., Grimley, E. D., Schenk, T., Schroeder, U. \& LeBeau, J. M. On the structural origins of ferroelectricity in $\mathrm{HfO}_{2}$ thin films. Appl. Phys. Lett. 106, 162905 (2015).

22. LeBeau, J. M., D’Alfonso, A. J., Wright, N. J., Allen, L. J. \& Stemmer, S. Determining ferroelectric polarity at the nanoscale. Appl. Phys. Lett. 98, 052904 (2011)

23. Materlik, R., Künneth, C. \& Kersch, A. The origin of ferroelectricity in $\mathrm{H}_{\mathrm{f} 1}$ ${ }_{-\mathrm{x}} \mathrm{Zr}_{\mathrm{x}} \mathrm{O}_{2}$ : a computational investigation and a surface energy model. J. Appl. Phys. 117, 134109 (2015).

24. Lee, W. et al. High current density and nonlinearity combination of selection device based on $\mathrm{TaO}_{\mathrm{x}} / \mathrm{TiO}_{2} / \mathrm{TaO}_{\mathrm{x}}$ structure for one selector-one resistor arrays. ACS Nano 6, 8166-8172 (2012).

25. Zhou, J., Kim, K. \& Lu, W. Crossbar RRAM arrays: selector device requirements during read operation. IEEE T Electron Dev. 61, 1369-1376 (2014).

26. Luo, Q. et al. Highly uniform and nonlinear selection device based on trapezoidal band structure for high density nano-crossbar memory array. Nano Res. 10, 3295-3302 (2017).

27. Vandelli, L. et al. Role of holes and electrons during erase of TANOS memories: evidences for dipole formation and its impact on reliability, in 2010 IEEE International Reliability Physics Symposium (IRPS) 731-737 (2010).

28. Higashi, Y. et al. New insights into the imprint effect in $\mathrm{FE}-\mathrm{HfO}_{2}$ and its recovery. in 2019 IEEE International Reliability Physics Symposium (IRPS) 1-7 (2019).

29. Florent, $\mathrm{K}$. et al. Investigation of the endurance of $\mathrm{FE}-\mathrm{HfO}_{2}$ devices by means of TDDB studies. in 2018 IEEE International Reliability Physics Symposium (IRPS) 6D.3-1-6D.3-7 (2018)

30. Padovani, A. et al. A comprehensive understanding of the erase of TANOS memories through charge separation experiments and simulations. IEEE T Electron Dev. 58, 3147-3155 (2011).

31. Qiao, F. et al. Reliability comparison of ISSG oxide and HTO as tunnel Dielectric in 3-D-SONOS applications. IEEE Electron Dev. Lett. 34, 620-622 (2013).

32. Yoon, K. J., Bae, W., Jeong, D. K. \& Hwang, C. S. Comprehensive writing margin analysis and its application to stacked one diode-one memory device for high-density crossbar resistance switching random access memory. Adv. Electron. Mater. 2, 1600326 (2016).

33. Zheng, Z. et al. Real-time polarization switch characterization of $\mathrm{HfZrO}_{4}$ for negative capacitance field-effect transistor applications. IEEE Electron Dev. Lett. 39, 1469-1472 (2018).

34. Koch, C. T. Determination of Core Structure Periodicity and Point Defect Density along Dislocations (2002).

35. Suyama, R., Horiuchi, H. \& Kume, S. Structural refinements of $\mathrm{ZrO}_{2}$ and $\mathrm{HfO}_{2}$ treated at $600^{\circ}$ C. J. Ceram. Soc. Jpn. 95, 567 (1987).

36. Kisi, E. H., Howard, C. J. \& Hill, R. J. The crystal structure of orthorhombic zirconia in partially stabilized zirconia. J. Am. Ceram. Soc. 72, 1757-1760 (2010).

37. Ohtaka, O., Yamanaka, T. \& Kume, S. Synthesis and X-ray structural analysis by the rietveld method of orthorhombic hafnia. J. Ceram. Soc. Jpn. 99, 826-827 (2010).

38. Maksymovych, P. et al. Polarization control of electron tunneling into ferroelectric surfaces. Science 324, 1421-1425 (2009).

39. Pintilie, L., Stancu, V., Trupina, L. \& Pintilie, I. Ferroelectric schottky diode behavior from a $\mathrm{SrRuO}_{3}-\mathrm{Pb}\left(\mathrm{Zr}_{0.2} \mathrm{Ti}_{0.8}\right) \mathrm{O}_{3}$-Ta structure. Phys. Rev. B 82, 085319 (2010).

40. Pintilie, L., Vrejoiu, I., Hesse, D., LeRhun, G. \& Alexe, M. Ferroelectric polarization-leakage current relation in high quality epitaxial $\mathrm{Pb}(\mathrm{Zr}, \mathrm{Ti}) \mathrm{O}_{3}$ films. Phys. Rev. B 75, 104103 (2007).

41. Hong, $\mathrm{S}$. et al. Large resistive switching in ferroelectric $\mathrm{BiFeO}_{3}$ nano-island based switchable diodes. Adv. Mater. 25, 2339-2343 (2013)

\section{Acknowledgements}

The authors thank the valuable discussion from Prof. C.S. Hwang with Seoul National University, Prof. A.Q. Jiang with Fudan University, Prof. B. Chen with Zhejiang University, J.X. Zhang with Beijing Normal University, and Prof. C. Song with Tsinghua University. This work was supported in part by the MOST of China under Grants 2016YFA0203800, 2018YFA0701500, 2016YFA0201803, 2018YFB040163, and 2018YFB0407502, and in part by the National Natural Science Foundation of China under Grants 61834009 and 61821091, and the Strategic Priority Research Program of the Chinese Academy of Sciences under Grant No. XDB44000000. 


\section{Author contributions}

Q. Luo, H.L., and M.L. designed the experiments. Q. Luo carried out the electrical experiments. Y.C. carried out the STEM test. Y.Y., R.H., and Y.Z. helped with the analysis of the STEM results. J. Yang, B.Y., and Q.R. conducted the circuit simulation. W.W. did the first-principles calculation. R.C. and H.M. carried out the fabrication of the planar metal-ferroelectric-metal (MFM) capacitors. T.G., H.Y., J. Yu, X.X., P.Y., X.L., L.T., D.S., and Q. Liu helped with data analysis. Q. Luo, H.L., and M.L. prepared the paper. H.L. and M.L. supervised the research.

\section{Competing interests}

The authors declare no competing interests.

\section{Additional information}

Supplementary information is available for this paper at https://doi.org/10.1038/s41467020-15159-2.

Correspondence and requests for materials should be addressed to H.L. or M.L.

Peer review information Nature Communications thanks Thomas Mikolajick, Fabrizio Torricelli and the other, anonymous, reviewer for their contribution to the peer review of this work.
Reprints and permission information is available at http://www.nature.com/reprints

Publisher's note Springer Nature remains neutral with regard to jurisdictional claims in published maps and institutional affiliations.

\section{(c) (i)}

Open Access This article is licensed under a Creative Commons Attribution 4.0 International License, which permits use, sharing, adaptation, distribution and reproduction in any medium or format, as long as you give appropriate credit to the original author(s) and the source, provide a link to the Creative Commons license, and indicate if changes were made. The images or other third party material in this article are included in the article's Creative Commons license, unless indicated otherwise in a credit line to the material. If material is not included in the article's Creative Commons license and your intended use is not permitted by statutory regulation or exceeds the permitted use, you will need to obtain permission directly from the copyright holder. To view a copy of this license, visit http://creativecommons.org/ licenses/by/4.0/.

(C) The Author(s) 2020 